\title{
CANONICAL HAMILTONIAN GUIDING CENTER VARIABLES
}

\author{
Roscoe B. White \\ Princeton Plasma Physies Laboratory \\ Princeton, New Jersey 08543
}

\begin{abstract}
A simplification of the canonical Hamiltonian variables for the guiding center motion of a charged particle in a general toroidal field is obtained using the Lagrangian formalism of Littlejohn.
\end{abstract}

\section{DISCLAIMER}

This repont was prepared as an socount of work spousosed by an areacy of the United Stales Governinent. Neitber the United States Covernmest nor any areacy thereor, nor any of their employeer, makes any warranty, expreas or implied, of anumes any keal liability or responsibithy for the accuracy, completeness, of unefuless of ay isformation, apparalus, product, of process disclosed, or represents that its use woukd not infringe privately owaed rights. Refer. ence herein to any specilic commercial product, process, or servioe by trade name, tradematt, manufacturer, of otherwise does not necessrily constilute or imply its eodorsement, recommendation, or favorinz by the Unicod States Comernment of any azency theroor. The views and opinions of authors expressed berein do not secescarily state or sellect those of the United States Goverement of any agency the reor. 
Hamiltonian guiding center calculation of particle movion in general toroidal fields is an effective way to examine long-time particle confinement and induced particle losses due to static magnetic perturbations or magnetohydrodynamic fluctuations. ${ }^{1,2}$ The expansion parameter for the derivation of equations of motion is the ratio of gyroradius to system size, $\rho$. Recently, the derivation of the canonical variables for a given magnetic field representation has been considerably simplified by an elegant Lagrangian formalism due to Litulejohn. ${ }^{3-5}$ However, for a general magnetic field the canonical variables are algebraically complicated due to nonorthogonality of the magnetic coordinates. In this note we show that this complexity can be removed by a modification of the parallel velocity which is of second order in $\rho$. This simplification of the canonical variables, of use in the study of guiding center motion, is also of interest for the formulation of gyrokinetics in general toroidal fields."

First consider magnetic configurations with toroidal symmetry and exact magnetic surfaces. Cases of broken symmetry are treated using perturbation theory.

In a general magnetic configuration possessing nested toroidal magnetic surfaces the field can be written, ${ }^{2}$ in contravariant and covariant form, as

$$
\begin{gathered}
\mathbf{B}=\nabla \psi \times \nabla \theta-\nabla \psi_{p} \times \nabla \zeta, \\
\mathbf{B}=g\left(\psi_{p}\right) \nabla \zeta+I\left(\psi_{p}\right) \nabla \theta+\delta\left(\psi_{p}, \theta\right) \nabla \psi_{p} .
\end{gathered}
$$

The magnetic coordinares $\psi, \psi_{p}, \zeta, 0$ are, respectively, toroidal and poloidal flux and toroidal and poloidal angies. The functions $g$ and $I$ are related to the 
poloidal and toroidal current, and $\delta$ describes the degree of nonorihogonality of the coordinate system. The field lines are straight in the $\zeta, \theta$ plane, i.e., $d \psi / d \psi_{p}=q\left(\psi_{p}\right)$. The fact that $I$ is a function of $\psi_{p}$ only is the result of a special choice for the Jacobian $J=\nabla \psi_{p} \cdot(\nabla \theta \times \nabla \zeta)$, with $B^{2} J=I+g q$.

Derivation of canonical variables is facilitated by the use of the guiding center Lagrangian due to Littlejoan, ${ }^{3,4}$ which has the form

$$
L=A^{*} \cdot \mathbf{v}+\mu \dot{\xi}-\mathbf{H}
$$

with $\mathbf{v}$ the guiding center velocity, $A^{*}=A+\rho_{\|} B$ the modified vector potential, $p_{\|}=v_{\|} / B$ the normalized parallel velocity, $\mu$ the magnetic moment, $\xi$ the gyrophrase and

$$
H=\frac{\rho_{\|}^{2} B^{2}}{2}+\mu B+\Phi,
$$

the Hamiltonian. The field magnitude $B$ is a function of $\psi$, and $\theta$ only, with $\zeta$ an ignorable coordinate. We use units defined by the on-axis gyrofrequency (time) and the major radius (distance). Originally, canonical Hamiltonian variables were found ${ }^{1,2}$ by the rather laborious procedure of first finding the time variation, following a particle orbit, of the variables $\psi, \theta, \zeta, \rho \|$ by using the guiding center motion $v$, e.g., $\dot{\psi}=\mathbf{v} \cdot \nabla \psi$. Here and in the following all quartities are functions of the location, i.e., of $\psi_{p}, \theta, \zeta$ only. The time derivative refers only to the change following a particle orbit. A convenient form for the guiding center motion, due to Morozov and Soloviev, ${ }^{7}$ is

$$
\mathbf{v}=\rho_{\|}\left(\mathbf{B}+\nabla \times \rho_{\|} \mathbf{B}\right) /\left(\mathbf{1}+\rho_{\|} \tilde{\mathbf{b}} \cdot \nabla \times \overline{\mathbf{b}}\right)
$$


with $\hat{b}=\mathbf{B} / B$, where in this equation $\rho_{\|}$is considered a function of position through Eq. (4) with $H$ replaced by the energy, and the denominator is a correction to the parallel drift discussed by several authors. ${ }^{8-11}$ This second order parallel drift correction is introduced strictly to preserve the Hamiltonian character of the motion. There are other second orier terms to $\mathrm{v}$, neglected to preserve Hamiltonian character order by order. Canonical variables were then found by writing the obtained expressions for $\dot{\psi}, \dot{\theta}, \dot{\zeta}, \dot{p}_{\|}$in terms of partial derivatives of the Hamiltonian, and finding the Darboux transformation to put the equations in canonical form. Littlejohn ${ }^{3}$ subsequently pointed out that a Lagrangian formalism made this procedure much less arduous, and significantly more transparent. We will see that the Lagrangian formalism also allows for the simplification of the canonical variables through a redefinition of the guiding center.

Canonical variables are obtained by substituting expressions for $A$ and $B$ in terms of magnetic coordinates into Eq. (3), and rewriting it in the form

$$
L=\sum_{i} P_{i} \dot{q}_{i}-H .
$$

If this form can be obtained, the canonical momenta and coordinates are immediately identified.

Rewriting Eq. (1) in the form $\mathbf{B}=\nabla \times\left(v \nabla \theta-\psi_{p} \nabla \zeta\right)=\nabla \times \mathbf{A}$ and substituting $A$ and $B$ into the Lagrangian, we find

$$
\begin{aligned}
L= & \left(\dot{\psi}+\rho_{\|\|} I\right) \dot{\theta}+\left(\rho_{\|} g-\psi_{p}\right) \dot{\zeta} \\
& +\mu \dot{\xi}-H+\delta \rho_{\|\|} \dot{\psi}_{p} .
\end{aligned}
$$


Except for the last term, this has the form given by Eq. (6). However, it can be put in canonical form. The appropriate modification, as found in Ref. 2 , is given by using the poloidal variable $\theta_{c}=\theta-p_{\|\|} \delta / q$. Substiuting $\dot{\theta}=\dot{\theta}_{c}+$ $(d / d t)\left(\rho_{\mid 1} \delta / q\right)$ into Eq. (7) and discarding the exact differential $(d / d t)\left(\rho_{\|} \delta \psi / q\right)$ as well as a term of order $\rho^{3}$, we are left with $L=P_{\theta} \dot{\theta}_{c}+P_{\zeta} \dot{\zeta}+\mu \dot{\xi}-H$, with

$$
\begin{aligned}
& P_{b}=\psi+\rho_{\|} I \\
& P_{6}=\rho_{\|} g-\psi_{p} .
\end{aligned}
$$

Note that these expressions are immediately recognizable as the covariant components of the sum of particle momentum and vector porential, just as in classical electrodynamics. Explicit equations of motion are readily obtained. First note from Eq. (8) that $\psi_{p}$ and $\rho_{\|}$are functions of $P_{b}, P_{6}$ only, and that

$$
\begin{array}{ll}
\frac{\partial \psi_{p}}{\partial P_{\theta}}=\frac{g}{D} & \frac{\partial \psi_{p}}{\partial P_{\zeta}}=\frac{-I}{D} \\
\frac{\partial \rho_{\|}}{\partial P_{\ell}}=\frac{1-\rho_{\|} g^{\prime}}{D} & \frac{\partial \rho_{\|}}{\partial P_{\zeta}}=\frac{q+\rho_{\|} I^{\prime}}{D}
\end{array}
$$

with $D=g q+I+\rho_{\|}\left[g I^{\prime}-I g^{\prime}\right]$, and primes indicate differentiation with respect to $\psi_{\mathrm{p}}$. It is then straightforward to find the explicit form of the equations of motion from $\dot{P}_{\zeta}=-\partial H / \partial \zeta, \dot{P}_{\theta}=-\partial H / \partial \theta, \dot{\theta}_{c}=\partial H / \partial P_{\theta}, \dot{\zeta}=\partial H / \partial P_{\zeta}$. For simplicity we set the electrostatic porential $\Phi$ equal to zero. The equations of motion take the form, to second order in $\rho$

$$
\dot{\zeta}=\frac{\rho_{\|} B^{2}\left(q+\rho_{\|} I^{\prime}\right)}{D}-\left(\mu+\rho_{\|}^{2} B\right) \frac{\partial B}{\partial \dot{\psi}_{p}} \frac{I}{D}+\left(\mu+\rho_{\|}^{2} B\right) \frac{\partial B}{\partial \theta} \frac{\delta}{D},
$$




$$
\begin{gathered}
\dot{\theta}_{c}=\frac{\rho_{\|\|} B^{2}\left(1-\rho_{\|} g^{\prime}\right)}{D}+\left(\mu+\rho_{\|}^{2} B\right) \frac{\partial B}{\partial \psi_{\mathrm{p}}} \frac{g}{D}+\left(\mu+\rho_{\|}^{2} B\right) \frac{\partial B}{\partial \theta} \frac{\delta}{D q}, \\
\dot{\psi}_{p}=\frac{-g}{D}\left(\mu+\rho_{\|}^{2} B\right) \frac{\partial B}{\partial \theta}, \\
\dot{\rho} \|_{\|}=-\frac{\left(\mu+\rho_{\|}^{2} B\right)}{D}\left(1-\rho_{\|} g^{\prime}\right) \frac{\partial B}{\partial \theta},
\end{gathered}
$$

where we have used the definition of $\theta_{c}$ to find that within corrections of higher onder in $\rho$

$$
\left.\frac{\partial \theta}{\partial P_{s}}\right|_{\theta_{c}, P_{G}, \zeta}=\frac{\delta}{q D} \quad,\left.\quad \frac{\partial \theta}{\partial P_{\zeta}}\right|_{P_{c}, P_{\theta}, \zeta}=\frac{\delta}{D} .
$$

Equations (10-13) clearly describe Hamiltonion motion. Directly calculating $v \cdot \nabla \theta, v \cdot \nabla \zeta$ by substituting Eqs. $(1,2)$ into Eq. (5) and using

$$
\hat{b} \cdot \nabla \times \hat{b}=\frac{g I^{\prime}-I g^{\prime}-g \partial \delta / \partial \theta}{g q+I},
$$

we find that $v$ given by Eq. (5) differs from the Hamiltonian motion by a second order term. Second order terms in the velocity can be modified by the addition of exact derivatives. For example, the term

$$
\frac{d}{d t}\left(\rho_{\|} \dot{o}\right) \approx \frac{\rho_{\|}^{2} B^{2}}{B} \frac{\partial \delta}{\partial \theta}-\frac{\delta}{D}\left(\mu+\rho_{\|}^{2} B\right) \frac{\partial B}{\partial \theta},
$$

where terms of higher order in $\rho$ have been discarded, if added to Eq. (10) eliminates the term in $\delta$ and adds a $\partial \delta / \partial \theta$ term almost equal to that given by Eq. (15). Within the guiding center description such a modification of the velocity is not significant. It produçes nonsecular, periodic motion of the magnitude of the gyroradius. This suggests that simplification of the Hamiltonian variables can be obtained by the addition of such terms. 
The Lagrangian formalism can be used to retain the variables $\theta$ and $\zeta$ as canonical, eliminating the term containing $\delta$ in Eq. (7) through a modification of the second order parallel guiding center drift. Return to Eq. (3) and replace the guiding center velocity $\mathbf{v}$ by $\mathbf{v}+w$, with $\mathbf{v}$ describing the guiding center motion, i.e., $v \cdot \nabla \theta=\dot{\theta}$. This adds a new term to Eq. (7) of the form $\mathrm{A}^{*} \cdot \mathbf{w}$, and we choose $w$ so that

$$
A^{*} \cdot w=-\delta p_{\|} \dot{\psi}_{p}
$$

First note that a change in gauge $\mathbf{A} \rightarrow \mathbf{A}+\nabla G$ adds an exacr derivative to L. Without loss of generality we choose the gauge $\mathbf{B} \cdot \mathbf{A}=0$. Noting from Eqs. (12) and (13) that to leading order $\dot{\psi}_{p}=g \dot{\rho}_{\|}$, we find

$$
\mathbf{w}=-\frac{\delta \dot{p}_{1} g \mathbf{B}}{B^{2}} \text {. }
$$

With this change the canonical variables become $\theta, P_{\theta}, \zeta, P_{\zeta}$ and the terms proportional to $\delta$ are eliminated from Eqs. (10) and (11). Directly from the Hamiltonian we find

$$
\begin{gathered}
\dot{\zeta}=\frac{\rho_{\|\|} B^{2}}{D}\left(q+\rho_{\|} I^{\prime}\right)-\left(\mu+\rho_{\|}^{2} B\right) \frac{I}{D} \frac{\partial B}{\partial \psi_{p}}, \\
\dot{\theta}=\frac{\rho_{\|} B^{2}}{D}\left(1-\rho_{\|} g^{\prime}\right)+\left(\mu+\rho_{\|}^{2} B\right) \frac{g}{D} \frac{\partial B}{\partial \psi_{p}}, \\
\dot{\psi}_{p}=\frac{-g}{D}\left(\mu+\rho_{\|}^{2} B\right) \frac{\partial B}{\partial \theta}, \\
\dot{\rho}_{\|}=-\frac{\left(\mu+\rho_{\|}^{2} B\right)}{D}\left(1-\rho_{\|} g^{\prime}\right) \frac{\partial B}{\partial \theta} .
\end{gathered}
$$


Discarding an exact derivative, we find

$$
\mathrm{w} \cdot \nabla \theta=\rho_{\|} \frac{d}{d t}\left(\frac{g \delta}{D}\right) \approx \frac{\rho_{\|}^{2} B^{2}}{D^{2}} g \frac{\partial \delta}{\partial \theta} .
$$

Similarly discarding an exact derivative in $w \cdot \nabla \zeta$, we find $(v-w) \cdot \nabla \zeta=\dot{\zeta}$, $(v-w) \cdot \nabla \theta=\dot{\theta}$. Thus we have oblained simplified expressions for the motion, with the magnetic coondinates $\theta, \zeta$ as canonical Hamiltonian variables. The velocity differs from the usual expression, Eq. (5), only by the second order parallel velocity $w$, which is zero if the magnetic coordinates are orthogonal. Since the second order parallel velocity is introduced only to maintain the Hamiltonian form, this is not a significant difference. Note that the function $\delta\left(\dot{\psi}_{p}, \theta\right)$ has disappeared entirely from the equations of motion. All of the dependence of the description of the motion appears in the functions $g, q, I$, and $B$.

Now consider the addition of a general magnetic perturbation, describud by the vector porential

$$
\overline{\mathrm{A}}=\alpha \nabla \zeta+\beta \nabla \theta+\gamma \nabla \psi_{p}
$$

with $\alpha, \beta, \gamma$ general functions of position. The Lagrangian then becomes $L=$ $\left(P_{\theta}+\beta\right) \dot{\theta}+\left(P_{6}+\alpha\right) \dot{\zeta}+\left(\delta \rho_{\|}+\gamma\right) \dot{\psi}_{p}+\mu \dot{\xi}-H$ with $P_{\theta}, P_{6}$ given by Eq. (8). Thus we identify the canonical variables as

$$
\begin{array}{ll}
\theta, & P_{\partial}^{\prime}=P_{\theta}+\beta\left(\psi_{p}, \theta, \zeta\right) \\
\zeta, & P_{\zeta}^{\prime}=P_{\zeta}+\alpha\left(\psi_{p}, \theta, \zeta\right)
\end{array}
$$

and again eliminate the term $\left(\delta \rho_{\|}+\uparrow\right) \dot{\psi}_{p}$ from the Lagrangian by a modification of the second order parallel velocity $\mathbf{w}$ with $\mathbf{A}^{*} \cdot \mathbf{w}=-\left(\delta \rho_{\|}+\gamma\right) \dot{\psi}_{p}$. We then 
find

$$
\mathbf{w}=-\frac{\left(\delta \rho_{\|}+\gamma\right) \dot{\psi}_{p} \mathbf{B}}{B^{2}}
$$

To find an expression for $w$, it is necessary to find the equations of motion to leading order. Note that, in general, in order to preserve the advantages arising from using a Hamiltonian formalism in numerical calculations of particle orbits, all orders in $\rho$ must be retained in the equations of motion. Truncation of these equations is useful only to calculate leading order effects.

The exact equations of motion in the perturbed field are readily found. We simply sketch the procedure here, since the results are algebraically very cumbersome, and for most applications a perturbed field of the restricted form $\tilde{\boldsymbol{A}}=\alpha \mathbf{B}$ is sufficient, and much easier to implement. ${ }^{1}$ From Eq. (25), using $\psi_{p}=\psi_{p}\left(P_{p}, P_{\zeta}\right)$ and Eq. (9) all of the partial derivatives of the transformation to the new canonical variables can be calculated. Finding the equations of motion is then an exercise in the use of the chain rule. We readily find, for example.

$$
\begin{aligned}
\dot{\psi}_{p}=\frac{-g}{D}\left(\rho_{\|}^{2} B+\mu\right) \frac{\partial B}{\partial \theta} & +\left[\frac{I}{D} \frac{\partial \alpha}{\partial \theta}-\frac{g}{D} \frac{\partial \beta}{\partial \theta}\right] \frac{\rho_{\|} B^{2}}{D} \\
+ & {\left[\frac{I}{D} \frac{\partial \alpha}{\partial \zeta}-\frac{g}{D} \frac{\partial \beta}{\partial \zeta}\right] \frac{\rho_{\|} B^{2} q}{D} }
\end{aligned}
$$

plus terms of order $\rho^{3}$.

Exact time derivatives of order $\rho^{2}$ discanded at various stages in these calculations integrate to zero by virtue of orbits closing in the $\psi_{p}, \theta$ plane, a property depending on the existence of the ignorable coordinate $\zeta$. This is no longer 
the case in the presence of field perturbations. If the perturbations are small, i.e., the orbits close except for small amounts $\Delta \theta, \Delta \psi_{p}$, then additional small changes are produced by these terms of order $\rho \Delta \theta, \rho \Delta \psi_{p}$. Motion across the drift surfaces produced by the neglected terms is smaller than that produced by the field perturbation by 2 factor $\rho$. If, however, the field is stochastic, then the initial guiding center coordinates do not uniquely determine the orbit. The motion is sensitive to the exact particle location, and depends for example on the initial gyrophase as well as the guiding center position. The inaccuracies given by discarding order $\rho^{2}$ exact derivatives are no larger than these initial condition inaccuracies. In such a system it makes litule sense to discuss precise individual particle orbits. Only statistical quantities obtained using a distribution of initial values are relevant, and thus these terms can also be safely ignored in this case.

\section{ACKNOWLEDGMENTS}

The author would like to thank Allen Boozer and Harold Weitzner for useful discussions. This work is supported by U.S. Department of Energy Contract No. DE-ACO2-76-CHO3073. 


\section{References}

'R.B. White, A.H. Boozer, and Ralph Hay, Phys. Fluids 25, 575 (1982).

${ }^{2}$ R.B. White and M.S. Chance, Phys. Fluids 27, 2455 (1984).

${ }^{3}$ R.G. Littlejohn, J. Plasma Physics 29, 111 (1983).

'R.G. Littlejohn, Physica Scripta T2/1, 119 (1982).

${ }^{5}$ R.G. Linlejohn, Phys. Fluids 28, 2015 (1985).

${ }^{6}$ P.J. Catio, W. M. Tang, and D.E. Baldwin, Plasma Phys. 23. 639 (1981).

${ }^{7}$ A.I Morozov and L.S. Soloviev, in Reviews of Plasma Physics (Consultants Bureau, New York, 1968), Vol. 2. translated from Russian; Voprosy Teorii Plazmy (Atomizdat, Moscow, 1963).

${ }^{8}$ R.G. Littlejohn, Phys. Fluids 24, 1730 (1981).

${ }^{9}$ A.H. Boozer, Phys. Fluids 23, 904 (1980).

${ }^{10}$ T.G. Northrop and J.A. Rome, Phys. Fluids 21, 384 (1978).

${ }^{11}$ R.D. Hazeltine, Plasma Phys. 15, 77 (1973). 